\title{
Linearization of planar homeomorphisms with a compact attractor.
}

\author{
Armengol Gasull $^{(a)}$, Jorge Groisman ${ }^{(b)}$ and Francesc Mañosas ${ }^{(a)}$
}

May 22, 2015

(a) Departament de Matemàtiques, Universitat Autònoma de Barcelona, 08193 Bellaterra, Barcelona, Spain (e-mail: gasull@mat.uab.cat, manyosas@mat.uab.cat)

(b) IMERL, Facultad de Ingeniería, Universidad de la República, Montevideo Uruguay (e-mail: jorgeg@fing.edu.uy)

\begin{abstract}
Kerékjártó's proved in 1934 that a planar homeomorphism with an asymptotically stable fixed point is conjugated, on its basin of attraction, to one of the maps $z \rightarrow z / 2$ or $z \rightarrow \bar{z} / 2$, according whether $f$ preserves or reverses the orientation. We extend this result to planar homeomorphisms having a compact attractor.
\end{abstract}

2000 MSC: Primary: 37C15. Secondary: 37C70, 54H20.

Keywords: Kerékjártó theorem, attractor, linearization, Lyapunov function

\section{Introduction.}

Consider the discrete dynamical system generated by a planar homeomorphism $f$. It is well known that if $f$ has an asymptotically stable fixed point, then its basin of attraction $\mathcal{U}$ is an open and simply connected subset of the plane. Moreover, it is proved by Kerékjártó $([7,8])$ that $f$ restricted to $\mathcal{U}$ is either conjugated to $L_{1}(z)=z / 2$ or $L_{2}(z)=\bar{z} / 2$ in $\mathbb{C}$, according whether $f$ preserves or reverses the orientation. A different proof of this result is also given in [4]. Afterwards, this result has been extended, with clear modifications, to $\mathbb{R}^{3}$ in [5] and to $\mathbb{R}^{m}$ for $m \neq 4,5$, in [6], when $f$ preserves orientation.

In this paper we will focus on the planar case and we extend Kerékjártó's result to the case where $f$ has a compact attractor. To state our result we need to introduce a new concept, the stabilizer of a compact attractor. This notion is analogous to the one proposed in [3] for ordinary differential equations.

Let $K$ be a compact attractor, not necessarily stable, and with basin of attraction $\mathcal{A}(K)$. Define the new compact set

$$
\widetilde{K}:=\{x \in \mathcal{A}(K): \alpha(x) \cap K \neq \emptyset\} .
$$


We call it the stabilizer of $K$. As we will see, $\widetilde{K}$ is a compact stable attractor with the same basin of attraction that $K$. Our main result is the following theorem:

Theorem A. Let $f: \mathbb{R}^{2} \rightarrow \mathbb{R}^{2}$ be a homeomorphism, let $K$ be a compact attractor and let $\mathcal{U}$ be its basin of attraction. Assume that $\mathcal{U}$ is connected and simply connected. Then $\mathcal{U} \backslash \widetilde{K}$ is homeomorphic to $\mathbb{R}^{2} \backslash\{\mathbf{0}\}$ and $\left.f\right|_{\mathcal{U} \backslash \widetilde{K}}$ is conjugated to $L_{1}(z)=z / 2$ or $L_{2}(z)=\bar{z} / 2$ on $\mathbb{R}^{2} \backslash\{\mathbf{0}\}$.

As corollaries of the above theorem, in Section 3 we get Kerékjártó's result and the following extension:

Corollary 1.1. Let $f: \mathbb{R}^{2} \rightarrow \mathbb{R}^{2}$ be a homeomorphism and let $K$ be a global compact attractor. Then $\mathbb{R}^{2} \backslash \widetilde{K}$ is homeomorphic to $\mathbb{R}^{2} \backslash\{\mathbf{0}\}$ and $\left.f\right|_{\mathbb{R}^{2} \backslash \widetilde{K}}$ is conjugated either to $L_{1}$ or to $L_{2}$ on $\mathbb{R}^{2} \backslash\{\mathbf{0}\}$.

Let us recall the main steps of Kerékjártó's proof. If $\gamma$ is a Jordan curve surrounding the fixed point, $p$, then clearly there exists an $n$ such that $f^{n}(\gamma)$ is also a Jordan curve, that surrounds $p$ and it is in the bounded component of $\mathcal{U} \backslash \gamma$. Then, using all the curves $f^{j}(\gamma), j=0,1, \ldots, n-1$, and some topological reasonings he constructs a new curve, say $\Gamma$, for which the same holds but with $n=1$. Then, the closed annulus $\mathcal{A}$, with boundaries $\Gamma$ and $f(\Gamma)$, constitutes a fundamental domain to construct the conjugacy $\psi$ between $f$ and $L_{j}, j=1$ or 2 . In fact, $\psi$ must send $\mathcal{A}$ to the set $A:=\{z \in \mathbb{C}: 1 / 2 \leq|z| \leq 1\}$, with some natural restrictions on the boundary. Then, this $\psi$ can be extended to $\mathcal{U}$ also in a natural way, by iteration.

Our prove Theorem A follows a similar approach, but with two main differences. The first one is that the curve $\Gamma$ having the property described above is constructed by using a different idea. Firstly, we prove the existence of a continuous Lyapunov function $L$ associated to the asymptotically stable compact set $\widetilde{K}$, by adapting a similar construction developped in [1] for ordinary differential equations. Afterwards, we show how to smooth some of the level sets of $L$ by using the Sard's theorem and the classification of the one dimensional manifolds. One of these smooth levels will be $\Gamma$. A second difference is that we use the extension of the Jordan's curve theorem, known as Schoenflies' theorem $([2,9])$, to prove the existence of a continuous conjugacy $\psi$ between the respective domains $\mathcal{A}$ and $A$, satisfying a suitable boundary condition.

In [10] J. Lewowicz proposed to use Lyapunov metrics (see next section for the precise definition) to study structural stability of homeomorphisms on compact manifolds, and similar concepts, such as topological stability and persistence. Since then, the method has been successfully applied to a wide spectrum of dynamical systems, such as hyperbolic and almost hyperbolic diffeomorphisms on manifolds, geodesic flows, pseudo-Anosov maps, billiards, expansive homeomorphisms on compact manifolds, in particular on surfaces, and on expansive homeomorphisms of the plane.

Our second result relates usual Lyapunov functions, Lyapunov metrics and global asymptotically stable fixed points in the plane.

Theorem B. Let $f: \mathbb{R}^{2} \longrightarrow \mathbb{R}^{2}$ be a homeomorphism and let $p$ be a fixed point. Then the following statements are equivalent: 
(i) $p$ is globally asymptotically stable.

(ii) There exists a Lyapunov function for $f$ and $p$.

(iii) $f$ is conjugate either to $L_{1}(z)=\frac{z}{2}$ or to $L_{2}(z)=\frac{\bar{z}}{2}$.

(iv) There exists a Lyapunov metric $D$ for which $p$ is D-stable.

In fact, by using the extension of Kerékjártó's theorem to dimension 3, or to dimensions $m>5$ in the preserving orientation case, the natural generalization of Theorem B to these settings also holds.

The paper is organized as follows. In Section 2 we introduce the stabilizer of an attractor, we give some examples and prove its basic properties in $\mathbb{R}^{m}$. We also prove some basic facts about the global asymptotic stable compacts and its relation with the existence of Lyapunov functions in $\mathbb{R}^{m}$ and, in this setting we also introduce some properties of the Lyapunov metrics. We conclude with some results valid in the planar case. In Section 3 we prove Theorems A and B and some corollaries, including Kerékjártó's theorem.

\section{General definitions and preliminary results}

We begin by listing some definitions and basic facts about the dynamics of homeomorphisms and we also introduce the stabilizer of an attractor compact set.

Let $X$ be a locally compact topological space and let $f: X \rightarrow X$ be a homeomorphism.

We say that $Y \subset X$ is invariant if $f(Y)=Y$. For $x \in X$ the omega limit set of $x$, denoted by $\omega(x)$, is the set of accumulation points of the sequence $\left\{f^{n}(x)\right\}_{n \in \mathbb{N}}$. Analogously the alpha limit set of $x$, denoted by $\alpha(x)$, is the set of accumulation points of the sequence $\left\{f^{-n}(x)\right\}_{n \in \mathbb{N}}$. The alpha and omega limit sets are closed and invariant subsets of $X$.

We will say that a compact set $K \subset X$ is an attractor if it is invariant and there exists a neighborhood $\mathcal{U}$ of $K$ such that for all $x \in \mathcal{U}, \omega(x) \neq \emptyset$ and $\omega(x) \subset K$. If $K$ is an attractor, the set $\mathcal{A}(K)=\{x \in X: \omega(x) \neq \emptyset$ and $\omega(x) \subset K\}$ is invariant and open and we call it the basin of attraction of $K$. In the case that $\mathcal{A}(K)=X$ we will say that $K$ is a global attractor.
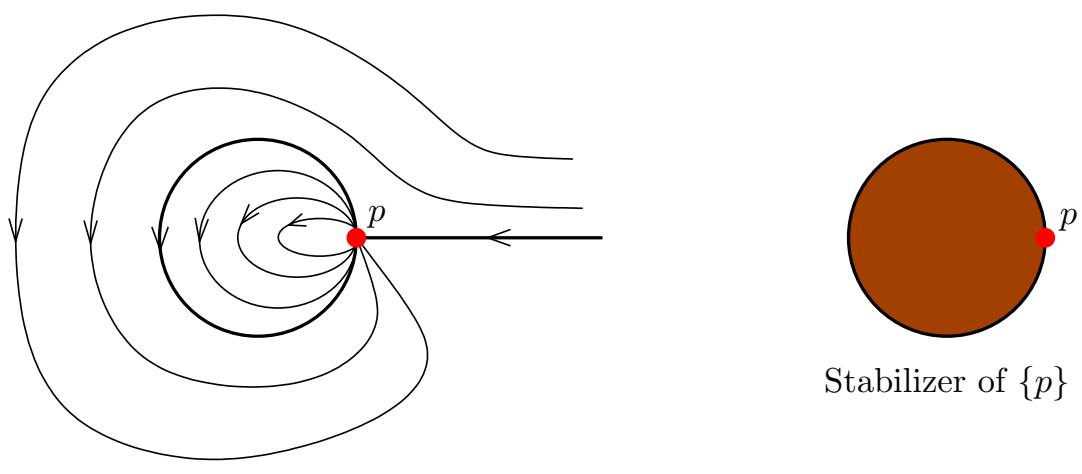

Stabilizer of $\{p\}$

Figure 1: Flow that defines $f_{1}$ and stabilizer of $\{p\}$. 


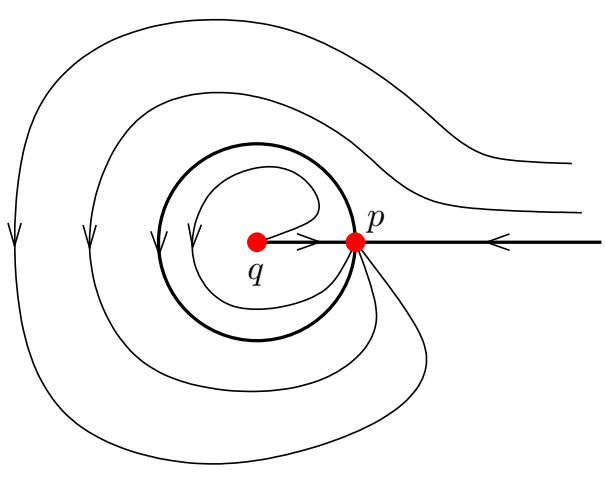

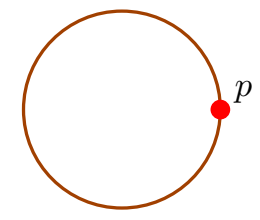

Stabilizer of $\{p\}$

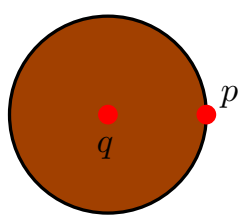

Stabilizer of $\{p, q\}$

Figure 2: Flow that defines $f_{2}$ and different stabilizers.

We will say that a compact set $K \subset X$ is asymptotically stable if it is an attractor and for all neighborhood $\mathcal{U}$ of $K$ there exists $\mathcal{V} \subset \mathcal{U}$ a neighborhood of $K$ such that $f^{n}(x) \in \mathcal{U}$ for all $x \in \mathcal{V}$ and $n \in \mathbb{N}$. In the case that $\mathcal{A}(K)=X$ we will say that $K$ is globally asymptotically stable.

Let $K$ be an attractor. Recall that we have introduced its stabilizer as $\widetilde{K}:=\{x \in$ $\mathcal{A}(K): \alpha(x) \cap K \neq \emptyset\}$. Let us show a couple of examples. Let $f_{1}$ and $f_{2}$ be the time one maps given by the flows with phase portraits given in Figures 1 and 2 , respectively. In these figures the stabilizers of different compact attractors are displayed. In particular, it is interesting to notice that for $f_{2}$, see Figure 2, when we consider $K=\{p\}$, then this set is a compact attractor, but Theorem A can not be applied because its basin of attraction $\mathcal{U}=\mathbb{R}^{2} \backslash\{q\}$, is not simply connected. On the other hand, taking $K=\{p, q\}$ we get a global attractor and by Corollary 1.1 , we obtain that, on $\mathbb{R}^{2} \backslash \widetilde{K}, f_{2}$ is conjugated with $L_{1}$.

Our first objective is to show that in our situation $\widetilde{K}$ is asymptotically stable with the same basin of $K$. We need some preliminary results.

From now on we restrict our attention to the case when $X$ is an open subset of $\mathbb{R}^{m}$. If $K$ is an attractor, we can always assume that $K$ is a global attractor by considering $\left.f\right|_{\mathcal{A}(K)}$ which is also a homeomorphism. If $\mathcal{U} \subset X$ we will denote by $\overline{\mathcal{U}}$ and $\mathcal{U}^{c}$ the closure of $\mathcal{U}$ and $X \backslash \mathcal{U}$, respectively

Lemma 2.1. Let $f: X \longrightarrow X$ be a homeomorphism where $X$ is an open subset of $\mathbb{R}^{m}$. Assume that $K \subset X$ is a global attractor. Let $Q_{1}$ be a compact neighborhood of $K$. Then there exists a compact neighborhood $Q_{2}$ such that $Q_{1} \subset Q_{2}$ and $f\left(Q_{2}\right) \subset Q_{2}$.

Proof. Let $x \in Q_{1}$ and $n_{x} \geq 1$ the first natural number such that $f^{n_{x}}(x) \in \operatorname{int}\left(Q_{1}\right)$. Let $B_{x}$ an open neighborhood of $x$ such that $\bar{B}_{x}$ is compact and $f^{n_{x}}\left(B_{x}\right) \subset \operatorname{int}\left(Q_{1}\right)$. Then $\left\{B_{x}\right\}_{x \in Q_{1}}$ is an open cover of $Q_{1}$. Let $x_{1}, \ldots, x_{l}$ such that $Q_{1} \subset \bigcup_{i=1}^{l} B_{x_{i}}$. Define

$$
Q_{2}=\bigcup_{i=1}^{l}\left(\bigcup_{j=0}^{n_{x_{i}}} f^{j}\left(\bar{B}_{x_{i}}\right)\right) .
$$

Clearly, $Q_{2}$ is a compact set and $f\left(Q_{2}\right) \subset Q_{2}$. 
Lemma 2.2. Set $X \subset \mathbb{R}^{m}$ open, let $f: X \longrightarrow X$ be a homeomorphism and let $K \subset X$ be a global attractor. Given any $x \in X$ we have that $x \in \widetilde{K}$ or $\alpha(x)=\emptyset$ (i.e. the negative orbit of $x$ leaves any compact set).

Proof. Let $x \in X$ and let $M \subset X$ be a compact set such that the negative orbit of $x$ does not leave $M$. Then $\alpha(x) \neq \emptyset$. Take $y \in \alpha(x)$. Then $w(y) \subset K$ and $w(y) \subset \alpha(x)$ (since $\alpha(x)$ is an invariant set). So $\alpha(x) \cap K \neq \emptyset$. Thus $x \in \widetilde{K}$.

Proposition 2.1. Set $X \subset \mathbb{R}^{m}$ open, let $f: X \longrightarrow X$ be a homeomorphism and let $K \subset X$ be an attractor. Then $\widetilde{K}$ is asymptotically stable with the same basin of attraction that $K$. Moreover $K$ is asymptotically stable if and only if $\widetilde{K}=K$.

Proof. We can restrict our attention to the case were $K$ is a global attractor. Notice that by definition $\widetilde{K}$ is invariant. Now, we prove that it is compact. By Lemma 2.1, there exists a compact neighborhood $Q$ of $K$ such that $f(Q) \subset Q$. Thus, $f^{-1}\left(Q^{c}\right) \subset Q^{c}$ and then $\widetilde{K} \subset Q$. Thus $\widetilde{K}$ is bounded. We will prove that $\widetilde{K}^{c}$ is an open set which will imply the compactness of $\widetilde{K}$. Let $p \in \widetilde{K}^{c}$. By Lemma 2.2, there exists $n \geq 0$ such that $f^{-n}(p) \in Q^{c}$. Let $\mathcal{V}$ be a neighborhood of $p$ such that $f^{-n}(\mathcal{V}) \subset Q^{c}$. Since $Q^{c}$ is invariant for $f^{-1}$, then $\alpha(x) \subset \bar{Q}^{c}$ for all $x \in \mathcal{V}$, so $\mathcal{V} \subset \widetilde{K}^{c}$. Then $\widetilde{K}$ is a compact set. By definition $K \subset \widetilde{K}$ so $\widetilde{K}$ is a global attractor. Let us see that $\widetilde{K}$ is asymptotically stable. Suppose that this is not true. Then there exists an open neighborhood $\mathcal{U}$ of $\widetilde{K}$ such that for all open neighborhood $\mathcal{V}$ of $\widetilde{K}$ there exist $x \in \mathcal{V}$ and $n_{x} \geq 0$ such that $f^{n_{x}}(x)$ does not belong to $\mathcal{U}$. Let $Q$ be the compact and positive invariant neighborhood of $\widetilde{K}$. Then there exists a sequence $\left\{x_{k}\right\}_{k}$ of $Q$ such that:

- $f^{n_{k}}\left(x_{k}\right)$ does not belong to $\mathcal{U}$ fore some $n_{k} \geq 0$,

- $\lim x_{k}=x \in \widetilde{K}$.

Since $x_{k} \in Q$ and $Q$ is a positive invariant set, we have that (taking a subsequence if it were necessary) $f^{n_{k}}\left(x_{k}\right)$ tends to a point $y \in Q \backslash \mathcal{U}$. By Lemma 2.2 there exist $j>0$ and a neighborhood $\mathcal{V}$ of $y$ such that $f^{-j}(y) \in Q^{c}$ and $f^{-j}(\mathcal{V}) \subset Q^{c}$. We claim that the sequence $\left(n_{k}\right)$ is unbounded. If not, we can take a subsequence $\left\{x_{k_{i}}\right\}_{i}$ such that tends to $x$ and $f^{n}\left(x_{k}\right) \rightarrow y$ for some constant $n$. Thus, $y=f^{n}(x)$ which contradicts the invariance of $\widetilde{K}$. So, let $k_{0} \in \mathbb{N}$ such that $f^{n_{k}}\left(x_{k}\right) \in \mathcal{V}$, for all $k>k_{0}$. Then $f^{n_{k}-j}\left(x_{k}\right) \in Q^{c}$, for all $k>k_{0}$, and since the sequence $\left(n_{k}\right)$ is unbounded there exists some $k$ such that $n_{k}-j>0$ which contradicts the positive invariance of $Q$. Therefore $\widetilde{K}$ is asymptotically stable. Since by definition $K \subset \widetilde{K}$ it follows that it is globally asymptotically stable. Thus the first statement holds. Clearly if $\widetilde{K}=K$, it follows that $K$ is asymptotically stable. Lastly assume that $K$ is asymptotically stable and we will see that $\widetilde{K}=K$. Suppose to arrive a contradiction that $x \in \widetilde{K} \backslash K$. Then since $\alpha(x) \cap K \neq \emptyset$ it follows that for any $\varepsilon$ the set $B_{\varepsilon}=\left\{y \in \mathbb{R}^{m}: d(y, K)<\varepsilon\right\}$ contains some pre-image of $x$ which contradicts the stability of $K$. This ends the proof of the Proposition.

\subsection{Lyapunov functions in $\mathbb{R}^{m}$}

A classical tool to investigate the stability of compact invariant sets is the existence of the so called Lyapunov functions. Let $f: \mathbb{R}^{m} \longrightarrow \mathbb{R}^{m}$ be a homeomorphism and $K \subset \mathbb{R}^{m}$ 
be a compact invariant set. We will say that a proper and continuous map $L: \mathbb{R}^{m} \longrightarrow \mathbb{R}$ is a Lyapunov function for $K$ and $f$, if it satisfies the following two properties:

(i) For all $x \in \mathbb{R}^{m}, L(x) \geq 0$ and $L(x)=0$ if and only if $x \in K$.

(ii) For all $x \in \mathbb{R}^{m} \backslash K, L(f(x))<L(x)$.

The following result relates the global asymptotical stability with the existence of Lyapunov functions.

Proposition 2.2. Let $f: \mathbb{R}^{m} \rightarrow \mathbb{R}^{m}$ be a homeomorphism and $K$ be a compact invariant subset. The the following statements are equivalent:

(a) There exists a Lyapunov function for $f$ and $K$.

(b) $K$ is globally asymptotically stable.

Proof. Assume that (a) holds and let $L: \mathbb{R}^{m} \longrightarrow \mathbb{R}$ be a Lyapunov function for $K$ and $f$. First we show that $K$ is a global atractor. Let $x \in \mathbb{R}^{m}$ and set $k=L(x)$. Then the positive orbit of $x$ is contained in $L^{-1}[0, k]$ which is a compact set. Therefore $\omega(x) \neq \emptyset$. We claim that $\omega(x) \subset K$. If not, there exists $y \in \omega(x)$ such that $L(y)=m \neq 0$. Therefore $L(f(y))=m^{\prime}<m$. Since $f(y) \in \omega(x)$ this implies that there exists a sequence $0<n_{1}<\ldots<n_{i} \ldots$ such that $\lim f^{n_{i}}(x)=f(y)$. In particular for $j$ large enough we will have $L\left(f^{n_{j}}(x)\right)=s<m$. Thus we will obtain that $f^{n}(x)<s<m=L(y)$ for $n>n_{j}$ that contradicts the fact that $y \in \omega(x)$. This proves the claim and shows that $K$ is a global atractor.

Now we show that $K$ is asymptotically stable. Let $U$ be an open neighborhood of $K$ and set $M=\min \{L(x) ; x \notin U\}$ that there exists because $L$ is proper. Moreover since $K \subset U$ it follows that $M \neq 0$. Assume to arrive a contradiction that for all $j>0$ there exists $x_{j}$ and $k_{j} \geq 0$ such that $d\left(x_{j}, K\right)<1 / j$ and $f^{k_{j}}\left(x_{j}\right) \notin U$. Thus we will obtain a sequence $x_{j}$ tending to $K$ with $L\left(x_{j}\right)>M$, which gives a contradiction with the continuity of $L$. Therefore there exists $m$ such that $B_{m}=\left\{x \in \mathbb{R}^{m} ; d(x, K)<1 / m\right\}$ satisfies that for all $y \in B_{m}$ and for all $r \geq 0, \quad f^{r}(y) \in U$. This ends the proof of the first implication.

Now assume that $K$ is globally asymptotically stable. Define $s: \mathbb{R}^{m} \longrightarrow \mathbb{R}$ by $s(x)=$ $d(x, K)=\min \{\|x-y\| ; y \in K\}$ which clearly is a positive continuous function that only vanishes at $K$. Since $K$ is is globally asymptotically stable for any $x \in \mathbb{R}^{m} \backslash K$ there exists $k_{x} \in \mathbb{N}$ such that $s\left(f^{n}(x)\right)<s(x) / 2$ for all $n>k_{x}$. Thus

$$
\Phi(x)=\sup \left\{s\left(f^{n}(x)\right) ; n \in \mathbb{N}\right\}=\max \left\{s\left(f^{n}(x)\right) ; n \in\left\{0, \ldots, k_{x}\right\}\right\}
$$

is well defined. Clearly it is continuous and since $\Phi(x) \geq s(x)$ it is also proper. By definition $\Phi$ satisfies property (i) and also satisfies that $\Phi(f(x)) \leq \Phi(x)$ for all $x \in \mathbb{R}^{m}$. Now consider

$$
L(x):=\sum_{n=0}^{\infty} \frac{\Phi\left(f^{n}(x)\right)}{2^{n}} .
$$

Clearly this series converges uniformly on compact subsets of $\mathbb{R}^{m}$ and hence defines a continuous function on $\mathbb{R}^{m}$. Moreover since $L(x) \geq \Phi(x) \geq s(x)$ for all $x \in \mathbb{R}^{m}$ it follows that $L$ is proper and satisfies property (i). On the other hand $L(f(x))-L(x)=$ $\sum_{i=0}^{\infty} \frac{\Phi\left(f^{n+1}(x)\right)-\Phi\left(f^{n}(x)\right)}{2^{n}} \leq 0$ and $L(f(x))-L(x)=0$ if and only if $\Phi(x)=\Phi\left(f^{n}(x)\right)$ for all $n \in \mathbb{N}$ which implies that $x \in K$ 


\subsection{Lyapunov metrics in $\mathbb{R}^{m}$}

To formulate the notion of Lyapunov metric we need to introduce some notation. Given a homeomorphism $f: \mathbb{R}^{m} \longrightarrow \mathbb{R}^{m}$ and a continuos map $G: \mathbb{R}^{m} \times \mathbb{R}^{m} \longrightarrow \mathbb{R}$ we will denote by $\Delta(G)$ the map $\Delta(G): \mathbb{R}^{m} \times \mathbb{R}^{m} \longrightarrow \mathbb{R}$ defined by

$$
\Delta(G)(x, y)=G(f(x), f(y))-G(x, y) .
$$

We will denote by $\Delta^{2}(G)$ the map $\Delta(\Delta(G))$.

Let $f: \mathbb{R}^{m} \rightarrow \mathbb{R}^{m}$ be a homeomorphism. We will say that $D: \mathbb{R}^{m} \times \mathbb{R}^{m} \rightarrow \mathbb{R}$ is a Lyapunov metric for $f$ if it is continuous, vanishes only on the diagonal, and is such that both $D(x, y)$ and $\Delta^{2}(D)(x, y)$ are positive for $(x, y)$ whenever $x \neq y$.

Let $f: \mathbb{R}^{m} \rightarrow \mathbb{R}^{m}$ be a homeomorphism of the plane that admits a Lyapunov metric $D$. A fixed point $x \in \mathbb{R}^{m}$ is said $D$-stable if the map $D_{x}: \mathbb{R}^{m} \longrightarrow \mathbb{R}$ defined by $D_{x}(y)=$ $D(x, y)$ is proper and given any $k^{\prime}>0$ there exists $k>0$ such that $D\left(x, f^{m}(y)\right)<k^{\prime}$ for all $m \geq 0$, whenever $D(x, y)<k$.

Proposition 2.3. Let $f: \mathbb{R}^{m} \rightarrow \mathbb{R}^{m}$ be a homeomorphism, let $D: \mathbb{R}^{m} \times \mathbb{R}^{m} \longrightarrow \mathbb{R}$ be a Lyapunov metric for $f$ and let $p$ be a fixed point that is D-stable. Then $p$ is globally asymptotically stable.

Proof. We will show that the map $D_{p}$ is a Lyapunov function for $f$ and $p$. By hypothesis it is positive and proper and only vanishes at $p$. So only remains to show that $D_{p}(f(x))<$ $D_{p}(x)$ for all $x \in \mathbb{R}^{m}, x \neq p$. That is $\Delta(D)(x, p)<0$ for all $x \neq p$. Set

$$
\mathcal{W}:=\left\{x \in: \Delta(D)\left(f^{m}(x), p\right)<0, \text { for all } m \geq 0\right\} \cup\{p\} .
$$

We will show that $\mathcal{W}=\mathbb{R}^{m}$.

First of all we claim that there exists a neighborhood $\mathcal{U}$ of $p$, such that $\mathcal{U} \subset \mathcal{W}$. To prove this note that since

$$
\Delta(D)(f(x), p)=\Delta(D)(x, p)+\Delta^{2}(D)(x, p),
$$

we have that

$$
\Delta(D)(f(x), p)>\Delta(D)(x, p),
$$

and consequently for all $m>0$,

$$
\Delta(D)\left(f^{m}(x), p\right)>\Delta(D)(x, p) .
$$

Therefore we get

$$
D\left(f^{m}(x), p\right)=D(x, p)+\sum_{i=0}^{m-1} \Delta(D)\left(f^{i}(x), p\right)>D(x, p)+m \Delta(D)(x, p) .
$$

Thus if $\Delta(D)(x, p)>0$ we obtain that $\lim _{m \rightarrow \infty} D\left(f^{m}(x), p\right)=\infty$. We obtain the same conclusion when $\Delta(D)(x, p)=0$, simply observing that for $y=f(x)$ we will have $\Delta(D)(y, p)>0$. Lastly note that if $\Delta(D)\left(f^{j}(x), p\right) \geq 0$ then $\lim _{m \rightarrow \infty} D\left(f^{m}\left(f^{j}(x)\right), p\right)=$ $\infty$ and so $\lim _{m \rightarrow \infty} D\left(f^{m}(x), p\right)=\infty$. Now we prove the claim. If such neighborhood $\mathcal{U}$ 
does not exist this implies that there exists a sequence $\left\{x_{i}\right\}_{i}$ such that $\lim x_{i}=p$ and $\lim _{m \rightarrow \infty} D\left(f^{m}\left(x_{i}\right), p\right)=\infty$ for all $i$. This fact clearly contradicts the fact that $p$ is $D$ stable. Note that in particular we have also showed that $\mathcal{W}$ is invariant. Next we will show that $\mathcal{W}$ is closed. Let $\left\{x_{k}\right\}_{k}$ be a convergent sequence of $\mathcal{W}$ and let $x$ be its limit point. If $x$ does not belong to $\mathcal{W}$ then there exists $m \in \mathbb{N}$ such that $D\left(f^{m}(x), p\right)>0$. Therefore, there exists a neighborhood $\mathcal{B}$ of $f^{m}(x)$ such that $D(y, p)>0$ for all $y \in \mathcal{B}$. Thus for a sufficiently large $k$, we have that $f^{m}\left(x_{k}\right) \in \mathcal{B}$ which contradicts the fact that $x_{k} \in \mathcal{W}$.

Next step is to show that $D\left(f^{m}(x), p\right)$ tends to zero when $m$ grows to infinite and $x \in \mathcal{W}$. Let us suppose that there exists a point $x \in \mathcal{W}$ such that $D\left(f^{m}(x), p\right)$ does not converge to zero. Note that since $x \in \mathcal{W}$, the function $D_{p}$ is decreasing over the positive orbit of $x$. Then the sequence $\left\{D\left(f^{m}(x), p\right)\right\}_{m \geq 0}$ is bounded by $D(x, p)$. Since $D_{p}$ is a proper function we obtain that the set $\left\{f^{m}(x), m \geq 0\right\}$ is bounded and then there exists an accumulation point $q \neq p$. Since $\mathcal{W}$ is closed we have that $q \in \mathcal{W}$, so $D(f(q), p)<$ $D(q, p)$. This implies the existence of $n_{1}<n_{2}$ such that $D\left(f^{n_{1}}(x), p\right)<D\left(f^{n_{2}}(x), p\right)$ which contradicts that $x \in \mathcal{W}$.

Lastly we will prove that $\mathcal{W}=\mathbb{R}^{m}$. Only remains to show that $\mathcal{W}$ is open. Let $x$ be an arbitrarily point of $\mathcal{W}$. By the above observation we know that $\lim _{m \rightarrow \infty} D\left(f^{m}(x), p\right)=0$. We claim that $\lim _{m \rightarrow \infty} f^{m}(x)=p$. If not using the fact that $D_{p}$ is a proper function we have that $\left\{f^{m}(x)\right\}_{m \geq 0}$ must accumulate in a point $q \neq p$. So, there exists some subsequence $\left\{f^{m_{k}}(x)\right\}_{m_{k}}$ converging to $q$. Using the continuity of $D$, we have that $D\left(f^{m_{k}}(x), p\right)$ tends to $D(q, p)$ which is a positive number. This fact is in contradiction with $\lim _{m \rightarrow \infty} D\left(f^{m}(x), p\right)=0$. Then $\lim _{m \rightarrow \infty} f^{m}(x)=p$. Therefore, there exists $k$ such that $f^{k}(x) \in \mathcal{U}$. Let $\mathcal{V}$ be an open neighborhood of $f^{k}(x)$ contained in $\mathcal{U}$. Then $f^{-k}(\mathcal{V})$ is an open neighborhood of $x$ contained in $\mathcal{W}$. Therefore $\mathcal{W}$ is open and then $\mathcal{W}=\mathbb{R}^{m}$. Thus $D_{p}$ is a Lyapunov function for $f$ and $p$ and the result follows from Proposition 2.2

\subsection{The planar case}

In this section we fix our attention to $\mathbb{R}^{2}$. Given a Jordan curve $J \subset \mathbb{R}^{2}$ we denote by $\operatorname{int}(J)$ the bounded connected component of $\mathbb{R}^{2} \backslash J$. Given two Jordan curves $J_{1}$ and $J_{2}$ we will write $J_{1} \prec J_{2}$ if $J_{1} \subset \operatorname{int}\left(J_{2}\right)$. Note that if $J_{1} \prec J_{2}$, in particular $J_{1} \cap J_{2}=\emptyset$. Note also that if $J_{1} \prec J_{2}$ and $h: \mathbb{R}^{2} \longrightarrow \mathbb{R}^{2}$ is a homeomorphism then $h\left(J_{1}\right) \prec h\left(J_{2}\right)$. Thus $h^{n}\left(J_{1}\right) \prec h^{n}\left(J_{2}\right)$ for all $n \in \mathbb{Z}$. Next Lemma plays a crucial role in the proof of Theorem A.

Lemma 2.3. Let $f: \mathbb{R}^{2} \rightarrow \mathbb{R}^{2}$ be a homeomorphism and $K$ globally asymptotically stable. Then there exists an analytic Jordan curve $J$ with $K \subset \operatorname{int}(J)$ satisfying that $f(J) \prec J$ and $K \subset \operatorname{int}(f(J))$.

Proof. From Proposition 2.2 there exists a Lyapunov function $L: \mathbb{R}^{2} \longrightarrow \mathbb{R}$ continuous, proper and strictly decreasing over the orbits (not contained in $K$ ) of $f$. The first objective is to modify $L$ to obtain another Lyapunov function $\bar{L}$ which is analytic (in fact polynomial) in some open range of levels. First we note that given $b>0$ there exists $0<a<b$ such that $f\left(L^{-1}[a, b]\right) \cap L^{-1}[a, b]=\emptyset$. To do this consider $c<b$ and set

$$
\bar{a}=\max \left\{L(f(x)) ; x \in L^{-1}[c, b]\right\} .
$$


This value is well defined because $L$ is proper and $L$ and $f$ continuous, and clearly $\bar{a}<b$ because $L$ is a Lyapunov function. Then if $\bar{a}<c$ it suffices to choose $a=c$. Otherwise we can choose any $a \in(\bar{a}, b)$.

Now choose $0<a<b$ such that $M \doteq L^{-1}[a, b]$ satisfies that $M \cap f(M)=\emptyset$. Choose also $0<\varepsilon<\frac{b-a}{4}$ and denote by $M_{\varepsilon}=L^{-1}([a+\varepsilon, b-\varepsilon])$ and $M_{2 \varepsilon}=L^{-1}([a+2 \varepsilon, b-2 \varepsilon])$ which are compact sets because $L$ is proper. By the Stone-Wierstrass theorem there exists a polynomial map $P$ such that $\max \{|P(x)-L(x)| ; x \in M\}<\min (a, \varepsilon / 4)$. In particular $P(x)$ will be positive in $M$. Set also $\mathcal{U}=L^{-1}\left((a+\varepsilon / 2, b-\varepsilon / 2)\right.$ and $g: \mathbb{R}^{2} \longrightarrow[0,1]$ a $\mathcal{C}^{\infty}$ function such that $g(x)=1$ for all $x \in M_{\varepsilon}$ and $g(x)=0$ for all $x \notin \mathcal{U}$. Lastly define $\bar{L}=g P+(1-g) L$. Simple computations show that $\bar{L}$ satisfies the following properties:

(1) $\bar{L}(x)=P(x)$ when $x \in M_{\varepsilon}$ and $\bar{L}(x)=L(x)$ when $x \notin \mathcal{U}$.

(2) $\bar{L}^{-1}([a+2 \varepsilon, b-2 \varepsilon]) \subset M_{\varepsilon}$.

(3) $\bar{L}(x) \in[a, b]$ for all $x \in M$.

Now we claim that $\bar{L}$ is still a proper Lyapunov function. It is proper because it coincides with $L$ out of $M$. By construction it follows that $\bar{L}$ satisfies the property (i) of a Lyapunov function. Now we show that it is decreasing over the orbits. Set $x \notin K$ and we need to show that $\bar{L}(f(x))<\bar{L}(x)$. This is clear when $x$ and $f(x)$ do not belong to $M$ because in this case $L$ and $\bar{L}$ coincide. Suppose that $x \in M$. By construction, $f(x) \notin M$ and $\bar{L}(f(x))=L(f(x))<a$. On the other hand, by $(3), \bar{L}(x) \geq a$. Lastly suppose that $f(x) \in M$. Also by construction $x \notin M$ and $\bar{L}(x)=L(x)>b$. Also by (3) we have that $\bar{L}(f(x)) \in[a, b]$. This ends the proof of the claim.

Note also that from (1) and (2) it follows that $\bar{L}$ is polynomial in $\bar{L}^{-1}([a+2 \varepsilon, b-2 \varepsilon])$. By Sard's theorem we get that for almost all $z \in(a+2 \varepsilon, b-2 \varepsilon), \bar{L}^{-1}(z)$ is an analytic compact manifold. Now we choose $z \in(a+2 \varepsilon, b-2 \varepsilon)$ with this property and we get that $L^{-1}(z)$ is a finite union of Jordan curves. Since $\bar{L}$ is proper it follows $\bar{L}^{-1}(z)$ separes $K$ and a circle of radius $R$ for large enough. Thus at least one of the Jordan curves of $L^{-1}(z)$ must surround $K$. Denote one of them by $J$.

To end the proof of the lemma we will show that $f(J) \prec J$. First of all note that since $J$ surrounds $K, f(K)=K$ and $f$ is a homeomorphism we have that $f(J)$ also surrounds $K$. Furthermore since $L(f(x))<z$ for all $x \in J$ it follows that either $J \prec f(J)$ or $f(J) \prec J$. However if $J \prec f(J)$ we also get $f(J) \prec f^{2}(J)$ because $f$ is a homeomorphism and hence $J \prec f^{2}(J)$. Iterating this argument we will have $J \prec f^{n}(J)$ for all $n \in \mathbb{N}$ that contradicts the fact that $K$ is globally asymptotically stable. This ends the proof of the lemma.

We end this section stating the classical Schoenflies' theorem, see for instance [2, 9]. In fact, as we will see in the proof of Theorem A, which we will need is an extension of Schoenflies' result for homeomorphism between two annuli. To prove this fact we were inspired by the proof of Corollary 2.1 of [9].

Theorem 2.1. Let $J_{1}$ and $J_{2}$ be simple closed curves. Then any homeomorphism $h$ : $J_{1} \rightarrow J_{2}$ can be extended to give a homeomorphism of $\operatorname{int}\left(J_{1}\right) \cup J_{1}$ onto $\operatorname{int}\left(J_{2}\right) \cup J_{2}$. 


\section{Proof of Theorems A and B.}

Proof of Theorem $A$. Since $\mathcal{U}$ is connected and simply connected by the Riemann theorem it is homeomorphic to $\mathbb{R}^{2}$. Therefore we can restrict our attention to the case when $\mathcal{U}=\mathbb{R}^{2}$ namely when $K$ is a global attractor. From Proposition 2.1 , the set $\widetilde{K}$ is globally asymptotically stable and from Lemma 2.3 we know that there exists a Jordan curve $J$ surrounding $\widetilde{K}$ such that $f(J) \prec J$. First of all we claim that the ring determined by $J$ and $F(J)$ that is $J \cup(\operatorname{int}(J) \backslash \operatorname{int}(F(J)))$ is contained in $\mathbb{R}^{2} \backslash \widetilde{K}$. This is because if $z \in J \cup(\operatorname{int}(J) \backslash \operatorname{int}(F(J)))$ then $f^{-1}(z)$ belongs to the ring determined by $f^{-1}(J)$ and $J$. Therefore $f^{-1}(z) \notin \widetilde{K}$ and since $\widetilde{K}$ is invariant $z \notin \widetilde{K}$. So the claim is proved. For $i=1,2$ denote by

$$
S_{i}=\left\{z \in \mathbb{R}^{2} ;\|z\|=1 / i\right\}, S_{i}^{+}=\left\{(x, y) \in S_{i} ; y \geq 0\right\} S_{i}^{-}=\left\{(x, y) \in S_{i} ; y \leq 0\right\}
$$

and consider a homeomorphism $g_{1}: J \longrightarrow S_{1}$.

Denote by

$$
J^{+}=g_{1}^{-1}\left(S_{1}^{+}\right), J^{-}=g_{1}^{-1}\left(S_{1}^{-}\right), a=g_{1}^{-1}(-1,0), \quad b=g_{1}^{-1}(1,0)
$$

and consider two simple and disjoint paths $\gamma_{1}, \gamma_{2}$ contained in the annulus determined by $J$ and $f(J)$ joining $a$ with $f(a)$ and $b$ with $f(b)$. Denote also by $\widehat{J}$ the Jordan curve obtained by gluing $J^{+}, f\left(J^{+}\right), \gamma_{1}$ and $\gamma_{2}$. There are two possibilities. Either $\operatorname{int}(\widehat{J}) \cap \operatorname{int}(f(J))=\emptyset$ or $\operatorname{int}(f(J)) \subset \operatorname{int}(\widehat{J})$. In the first case consider homeomorphisms $g_{3}$ and $g_{4}$ from $\gamma_{1}$ to $I_{1}=\{(t, 0) ; t \in[-1,-1 / 2]\}$ and from $\gamma_{2}$ to $I_{2}=\{(t, 0) ; t \in[1 / 2,1]\}$ and $g_{2}: f(J) \longrightarrow S_{2}$ given by $g_{2}(x)=\frac{g_{1}\left(f^{-1}(x)\right)}{2}$. Gluing $g_{1}, g_{2}, g_{3}$ and $g_{4}$ we obtain and homeomorphism from $\widehat{J}$ to the Jordan curve formed by $S_{1}^{+}, S_{2}^{+}, I_{1}$ and $I_{2}$. Thus by the Schoenflies' theorem this homeomorphism can be extended to a homeomorphism $g^{+}$between the interiors of both Jordan curves, see Figure 3.

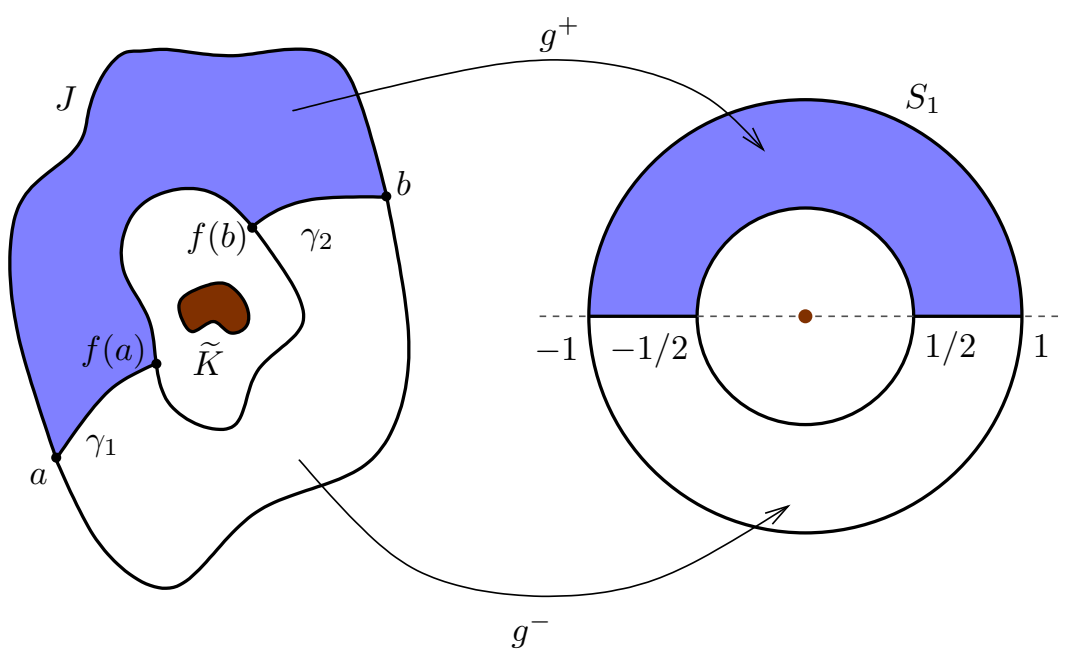

Figure 3: Construction of $g$ in the preserving orientation case.

Arguing similarly gluing $g_{1}, g_{2}, g_{3}$ and $g_{4}$ we obtain a homeomorphism between the Jordan curve formed by $J^{-}, f\left(J^{-}\right), \gamma_{1}, \gamma_{2}$ to the Jordan curve formed by $S_{1}^{-}, S_{2}^{-}, I_{1}$ and $I_{2}$. 
We also extend these homeomorphisms to a homeomorphism $g^{-}$between its interiors. Since $g^{+}$and $g^{-}$coincides in $\gamma_{1}$ and $\gamma_{2}$ we can glue both homeomorphisms to obtain a homeomorphism $g$ between the annulus determined by $J$ and $f(J)$ that we denote by $\mathcal{A}$ and the annulus determined by $S_{1}$ and $S_{2}$.

Now we claim that $\mathbb{R}^{2} \backslash \widetilde{K}=\cup_{i \in \mathbb{Z}} f^{i}(\mathcal{A})$. Let $z \in \mathbb{R}^{2} \backslash \widetilde{K}$ and assume that $z \notin \mathcal{A}$. If $z \in \cup_{i \in \mathbb{Z}} f^{n}(J)$ there is nothing to prove. From Lemma 2.2 it follows that there exists $i \leq 0$ such that $f^{i}(z) \notin \operatorname{int}(J)$. Now since $K$ is an attractor there exists a first $j>0$ such that $f^{i+j}(z) \in \operatorname{int}(J)$. This implies that $f^{(i+j)}(z) \in \mathcal{A}$ and hence $z \in f^{-(i+j)}(\mathcal{A})$.

Lastly let $h: \mathbb{R}^{2} \backslash \widetilde{K} \longrightarrow \mathbb{R}^{2} \backslash\{\mathbf{0}\}$ defined by $h(z)=2^{n} g\left(f^{n}(z)\right)$ when $f^{n}(z) \in \mathcal{A}$. Note that if $z \notin \cup_{n \in \mathbb{Z}} f^{n}(J)$ then the map $h$ is uniquely defined. If $z \in f^{n}(J)$ for some $n \in Z$ then $f^{-n}(z) \in J \subset \mathcal{A}$ and $f^{-n+1}(z) \in f(J) \subset \mathcal{A}$. Thus $h(z)=2^{-n} g\left(f^{-n}(z)\right)=2^{-n} g_{1}\left(f^{-n}(z)\right)$ or $h(z)=2^{-n+1} g\left(f^{-n+1}(z)\right)=2^{-n+1} g_{2}\left(f^{-n+1}(z)\right)=2^{-n+1} \frac{g_{1}\left(f^{-n}(z)\right)}{2}=2^{-n} g_{1}\left(f^{-n}(z)\right)$. Therefore $h$ is a well defined homeomorphism. Lastly we have $h(z)=2^{n} g\left(f^{n}(z)\right)=$ $2\left(2^{n-1} g\left(f^{n-1}(f(z))\right)\right)=2 h(f(z))$ which implies that $h\left(f\left(h^{-1}(z)\right)\right)=z / 2$. In particular $f$ preserves orientation. This ends the proof of the theorem in this case.

When $\operatorname{int}(f(J)) \subset \operatorname{int}(\tilde{J})$ the proof follows in a similar way considering the Jordan curve $J^{*}$ obtained joining $J^{+}, f\left(J^{-}\right), \gamma_{1}, \gamma_{2}$ and the map $g_{2}^{*}$ given by $g_{2}^{*}(x)=\frac{\overline{g_{1}\left(f^{-1}(x)\right)}}{2}$ instead of $\tilde{J}$ and $g_{2}$. In this case the corresponding map $h^{*}$ satisfies that $h^{*}\left(f\left(\left(h^{*}\right)^{-1}(z)\right)\right)=$ $\bar{z} / 2$ and hence $f$ reverses orientation. This ends the proof of the theorem.

The proof of Corollary 1.1 is straightforward from Theorem A because in this case the basin of attraction is the whole plane, that is $\mathcal{U}=\mathbb{R}^{2}$ and hence it is clearly is connected and simply connected. Next, we prove Kerékjártó's theorem.

Proof of Kerékjárto's theorem. We want to apply Theorem A when $K=\{p\}$, where $p$ is an asymptotically stable fixed point and $\mathcal{U}$ is its basin of attraction. Hence the result follows once we prove that $\mathcal{U}$ is connected and simply connected. This is so, because we obtain a homeomorphism $h: \mathcal{U} \backslash\{p\} \longrightarrow \mathbb{R}^{2} \backslash\{\mathbf{0}\}$ that linearizes $f$. Clearly this homeomorphism extends to a homeomorphism between $\mathcal{U}$ and $\mathbb{R}^{2}$ by putting $h(p)=\mathbf{0}$.

The above properties of $\mathcal{U}$ are well known, but we prove them for the sake of completeness. First, let us see that $\mathcal{U}$ is arc-connected. To do this let $D \subset \mathcal{U}$ be an open disc containing $p$ and $z_{1}, z_{2} \in \mathcal{U}$. Since $p$ is asymptotically stable there exists $n$ such that $f^{n}\left(z_{1}\right), f^{n}\left(z_{2}\right) \in D$. Now set $\alpha \subset D$ an arc joining $f^{n}\left(z_{1}\right)$ and $f^{n}\left(z_{2}\right)$. Then $\bar{\alpha}=f^{-n}(\gamma)$ is an arc joining $z_{1}$ and $z_{2}$. Since $f^{n}(\bar{\alpha}) \subset D \subset \mathcal{U}$ and $\mathcal{U}$ is $f$-invariant it follows that $\bar{\alpha} \subset \mathcal{U}$.

Now we show that $\mathcal{U}$ is simply connected. Let $J \subset \mathcal{U}$ be a Jordan curve and we will see that $\operatorname{int}(J) \subset \mathcal{U}$. As before let $D \subset \mathcal{U}$ be an open disc containing $p$ and $n \in \mathbb{N}$ be such that $f^{n}(J) \subset D$. Since $f$ is a homeomorphism $f^{n}(J)$ is also a Jordan curve and $f^{n}(\operatorname{int}(J))=\operatorname{int}\left(f^{n}(J)\right) \subset D \subset \mathcal{U}$. Since $\mathcal{U}$ is $f$-invariant, $\operatorname{int}(J) \subset \mathcal{U}$ and the curve $J$ can be deformed in $\mathcal{U}$ to a point.

Collecting all our results in the plane we can prove Theorem B.

Proof of Theorem B. The equivalence (i) $\Leftrightarrow$ (ii) follows from Proposition 2.2 while the double implication (i) $\Leftrightarrow$ (iii) follows from Kerékjártó's theorem. Also statement (iv) implies statement (i) from Proposition 2.3. So to finish the proof it suffices to show that statement (iii) implies (iv). This is a consequence of the fact that the usual euclidian 
metric $D$ is a Lyapunov metric for $L_{1}$ and $L_{2}$ for which $(0,0)$ is $D$-stable. Then the transported metric via the conjugation gives the desired Lyapunov metric for $f$.

\section{Acknowledgments}

We thank Rafael Ortega for sending us his interesting paper [11], from which we learnt about Kerékjártó's result and its extensions.

The first author is supported by a MINECO grant number MTM2013-40998-P and by a CIRIT grant number 2014SGR568. The third author by a MINECO grant number MTM2014-52209-C2-1-P.

\section{References}

[1] N.P. Bhatia and G. P. Szego, "Stability theory of dynamical systems." Reprint of the 1970 original. Classics in Mathematics. Springer-Verlag, Berlin, 2002.

[2] S. S. Cairns, An elementary proof of the Jordan-Schoenflies theorem, Proc. Amer. Math. Soc. 2, (1951) 860-867.

[3] A. Cima, F. Mañosas, J. Villadelprat, A Poincaré-Hopf theorem for noncompact manifolds, Topology, 37 (1998) 261-277.

[4] T. Homma and S. Kinoshita, On the regularity of homeomorphisms of $E^{n}$, J. Math. Soc. Japan, 5 (1953) 365-371.

[5] T. Homma and S. Kinoshita, On a topological characterization of the dilatation in $E^{3}$, Osaka Math. J. 6 (1954), 135-144.

[6] L.S. Husch, A topological characterization of the dilation in $E^{n}$, Proc. Amer. Math. Soc., 28 (1971) 234-236.

[7] B. de Kerékjártó, Sur le caractère topologique des représentations conformes, C. R. Acad. Sci. Paris, 198 (1934) 317-320. [In French].

[8] B. von Kerékjártó, Topologische Charakterisierung der linearen Abbildungen, Acta Litt. Acad. Sei. Szeged. 6 (1934), 235-262. [In German].

[9] B. Kolev, " Plane Topology and Dynamical Systems." École thématique. Summer School "Systèmes Dynamiques et Topologie en Petites Dimensions", Grenoble, France, 1994, pp.35. < cel-00719540 >

[10] J. Lewowicz, Lyapunov functions and topological stability, J. Differential Equations 38 (1980), 192-209.

[11] P. Ortega and F. R. Ruiz del Portal, Attractors with vanishing rotation number, J. Eur. Math. Soc. (JEMS), 13 (2011), 1569-1590. 